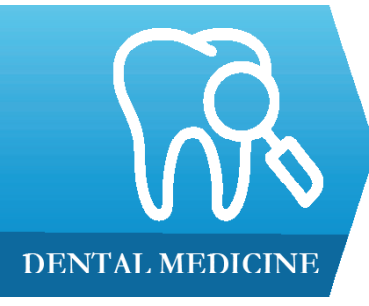

1) Department of Oral and Maxillofacial Pathology, Goenka Research Institute of Dental Science, Gandhinagar, Gujarat, India

2) Department of Oral and Maxillofacial Pathology, Index College of Dental Science, Indore, Madhya Pradesh, India

3) Department of Oral and Maxillofacial Pathology, Goenka Research Institute of Dental Science, Gandhinagar, Gujarat, India.

4) Department of Oral Pathology, K.M. Shah Dental College, Sumandeep Vidyapeeth, Vadodara, Gujarat, India

DOI: $10.15386 / \mathrm{mpr}-1439$

Manuscript received: 05.08.2019

Received in revised form: 05.10.2019

Accepted: 21.10.2019

Address for correspondence:

editorjrad@gmail.com

This work is licensed under a Creative Commons Attribution-NonCommercialNoDerivatives 4.0 International License

\title{
Effect of tobacco in human oral leukoplakia: a cytomorphometric analysis
}

Uday Patel ${ }^{1}$, Rina $\mathrm{Shah}^{2}$, Alpesh $\mathrm{Patel}^{3}$, Shreyas Shah ${ }^{4}$, Dhruva Patel ${ }^{3}$, Anil Patel ${ }^{3}$

\begin{abstract}
Objectives. Tobacco use is one of the most critical risk factors for different oral diseases. The aim of this study is to demonstrate the effect of tobacco on oral mucosa by cytomorphometric analysis of cells with the help of exfoliative cytology and to find out the improvement in diagnostic sensitivity of exfoliative cytology in the detection of dysplastic changes and early oral malignancy.

Methods. The nuclear area (NA) and cytoplasmic area (CA) of cells were measured within cytological smear obtained from leukoplakia lesions of buccal mucosa of 90 tobacco users, 30 smokers (TS), 30 chewers (TC) and 30 with combined habit of smoking and chewing (TSC) and from normal buccal mucosa of 30 non users (NU) of tobacco. Each habit group consisted of 30 tobacco users with oral leukoplakia lesion with mild epithelial dysplasia only. The 30 non-users of tobacco served as controls. The mean values of the CA and NA were obtained for each case, and the nuclear/cytoplasmic area (NA/CA) ratio was calculated.

Results. The results showed a statistically significant increase $(\mathrm{P}<0.001)$ in mean NA and a statistically significant decrease $(\mathrm{P}<0.001)$ in mean CA values of tobacco users with leukoplakia as compared to non-users, hence NA/CA ratio value was significantly higher in tobacco users with the lesion.

Conclusion. The changes in cellular morphology caused by tobacco use can be visualized by use of exfoliative cytology with the help of cytomorphometric analysis. The evaluation of parameters (NA, CA and NA/CA ratio) may increase the sensitivity of exfoliative cytology for the early diagnosis of oral premalignant and malignant lesions.
\end{abstract}

Keywords: cytomorphometry, oral leukoplakia, oral exfoliative cytology, tobacco smoking and chewing

\section{Introduction}

Oral mucosa reflects the general health of the body. The oral mucosa however strong and elastic to perform various functions may still be subjected to scores of insults. Insults may come in the form of multiple habits like chewing pan, tobacco, betel nut, smoking, alcohol drinking, which with the passage of years, leave their marks on the oral mucosa. The most unhealthy and dangerous entities associated with these habits are the clutches of oral cancer [1].

Tobacco habits are practised in various different forms, and many of them are specific to some regions of India. A number of lesions have been reported among tobacco chewers and smokers [2]. All parts of the oral cavity are susceptible to cancer from tobacco smoking or chewing, including the lip, tongue, buccal mucosa and masticatory mucosa. The mouth is the only site which permits viewing with the naked eye the ravages of smoked and smokeless (chewing) tobacco [3].

Histological changes of the oral mucosa have been reported in association with tobacco use [4,5]. Tobacco consumption affects the epithelium of the oral mucosa and results in the appearance of tissue. This alteration in mucosa may lead to the thickening of the 
epithelium and increase in pigmentation. Tobacco has an irritating effect on the minor salivary gland and increases a person's risk for oral cancer. The changes taking place in the oral epithelium are responsible for alteration in the morphology of exfoliated cells. The exfoliated cells show tobacco-induced mucosal changes. These changes can be examined with the help of exfoliative cytological technique [6-9]. Cytodiagnosis that is the use of cytology in the diagnosis is an old method of diagnosis. Exfoliative cytology offers a simple, quick, painless, bloodless and noninvasive technique that can be repeated frequently with little discomfort to the patient [10].

The smear obtained by exfoliative cytology can be analyzed quantitatively and qualitatively [11]. With advancements in the field of quantitative oral exfoliative cytology, various parameters such as nuclear size, cell size, nuclear-to-cytoplasmic ratio, nuclear shape, nuclear discontinuity, optical density and nuclear texture can be evaluated collectively in order to establish the diagnosis of premalignant and malignant lesions [12-17]. Of these parameters, the nuclear and cytoplasmic area and the nuclear-to-cytoplasmic ratio have been shown to be significant in the diagnosis of oral lesions [18]. The variations obtained in these parameters have been attributed to exposure to carcinogenic agents like tobacco [13].

The alterations in the size of the cell and nuclei have been observed in premalignant and malignant lesions $[11,13]$. Taking this into consideration, the present study was carried out to assess the effect of tobacco smoking and tobacco chewing, on the oral mucosa and to compare the cytomorphology of cells collected from oral leukoplakia lesions of the oral mucosa of tobacco users with those of tobacco non-users.

\section{Methods}

The study included 2 groups: habit group [tobacco smoking (TS), tobacco chewing (TC), tobacco smoking and chewing (TSC)] andcControl group. All the subjects from both genders in the study groups practised tobacco habits for more than ten years. None of the group members was on any medication or treatment. Each habit group consisted of 30 tobacco users with oral leukoplakia lesions with mild epithelial dysplasia only, and biopsy was taken with written consent for each case for confirmation of clinical diagnosis. The oral epithelial dysplasia was diagnosed according to the WHO 2005 classification(19). The control group consisted of 30 non-users (NU) of tobacco, betel quid and alcohol.

The information about the habit (i.e. duration and frequency of smoking and chewing, overnight placement of tobacco quid inside the oral cavity and use of alcohol) was recorded for each individual in detail. All the individuals of any group were over 40 years of age. Alcoholic, diabetic and anemic individuals of any group were excluded from this study.
Following written consent, oral exfoliative cytological smears were prepared from lesions of buccal mucosa immediately before biopsy in the habit groups and from buccal mucosa in the non-users group. Patients were asked to rinse the oral cavity with normal saline. The oral mucosa was dried with gauze swab to remove surface debris and excess saliva. Smears were obtained using a gentle scraping motion exerting little pressure with a wooden spatula moistened with normal saline. The scraps were smeared onto the center of the glass slide and spread over a large area preventing clumping of cells. Then smears were fixed immediately in 95\% ethyl alcohol for 1 hour and stained with the Papanicolaou stain. The cytomorphometric analysis was determined using the Software (Dewinter Biowizard 4.1 image analysis system). The smears were placed on Labomed ${ }^{\mathrm{TM}}$ ATC2000 microscope, and the cells were projected onto the monitor via Sony W35 colour video camera at $\mathrm{x} 400$ magnification. All the examinations were performed by a single-blinded examiner.

Only clearly defined cells were measured, avoiding clumped or folded cells and unusually distorted nuclei and cells. One hundred cells were selected by systemic sampling in a stepwise manner, moving the microscope stage from left to right and then down and across in order to avoid measuring the same cell again. For cytoplasmic area (CA) and nuclear area (NA) measurement, the perimeter of the cell and nucleus was measured by drawing nuclear and cell boundaries using the digital cursor (Figure 1). The nuclear/ cytoplasmic area (NA/CA) ratio was calculated. Statistical analyses were carried out by the independent samples t-test, the least significant procedure (LSD) and analysis of variance (ANOVA) using SPSS (v16.0 for windows, IL, USA) software.

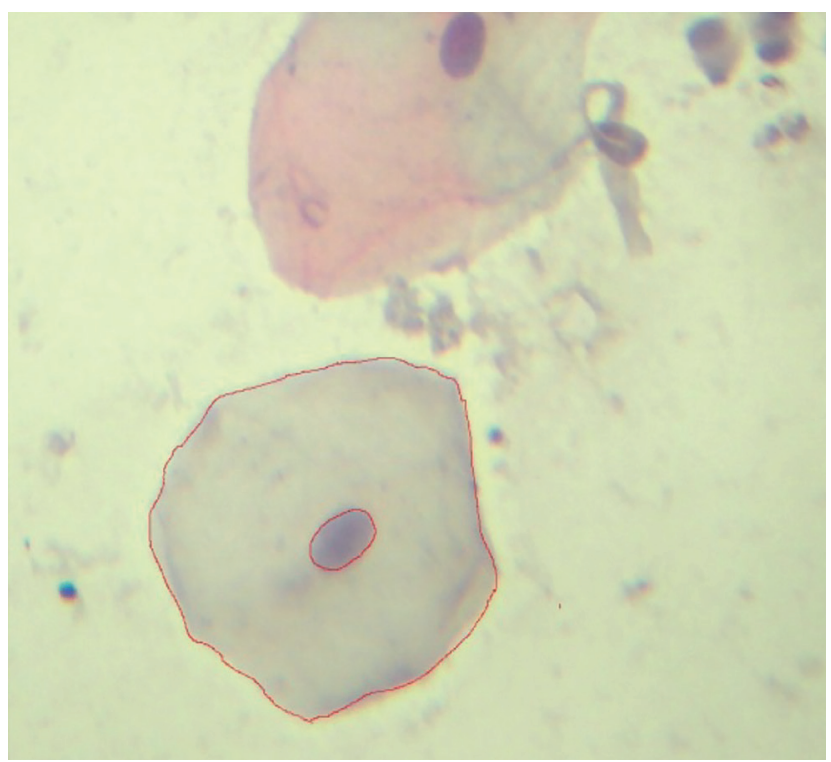

Figure 1. Photomicrograph showing tracing of cell area and nuclear area of a cell using image analysis software (Dewinter Biowizard 4.1) (Papanicolaou stain, 40X). 


\section{Results}

Distribution of study groups according to gender shows that tobacco smokers $(96.67 \%)$ in males were maximum in number followed by smoking and chewing group $(86.67 \%)$, chewing group $(70 \%)$, and non-users $(56.67 \%)$ whereas in females non-users outnumbered the tobacco users (Table I). All the subjects in the study groups practised tobacco habit for more than ten years. The CA, NA and NA/CA ratio values are plotted separately in the form of box plots (Figure 2). These illustrate the range of values observed and the box indicates the distribution of central $50 \%$ of data. The line within the box represents the median value, with the $25 \%$ of values above and below the median completing the box. There was a progressive reduction in mean $\mathrm{CA}$ from non-users to tobacco smokers through tobacco chewers to combined habit group (Table IIA). The NA was progressively increased from non-users to tobacco chewers through combine habit group to tobacco smokers (Table IIB).

Table I. Distribution of study groups according to gender.

\begin{tabular}{l|c|c|c} 
Study Group & Total No. of & \multicolumn{2}{|c}{ Sex } \\
\cline { 3 - 4 } samples & Male & Female \\
\hline Non users (NU) & 30 & 17 & 13 \\
& & $(56.67 \%)$ & $(43.33 \%)$ \\
Tobacco smoking (TS) & 30 & 29 & 1 \\
& & $(96.67 \%)$ & $(3.33 \%)$ \\
Tobacco chewing (TC) & 30 & 21 & 9 \\
$\begin{array}{l}\text { Tobacco smoking and } \\
\text { chewing (TSC) }\end{array}$ & 30 & $(70 \%)$ & $(30 \%)$ \\
& & 26 & 4 \\
& & $(86.67 \%)$ & $(13.33 \%)$
\end{tabular}

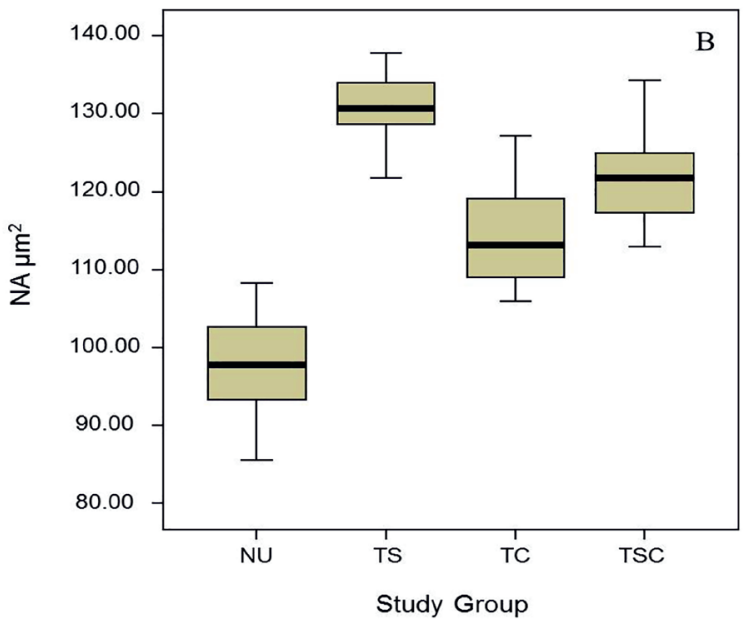

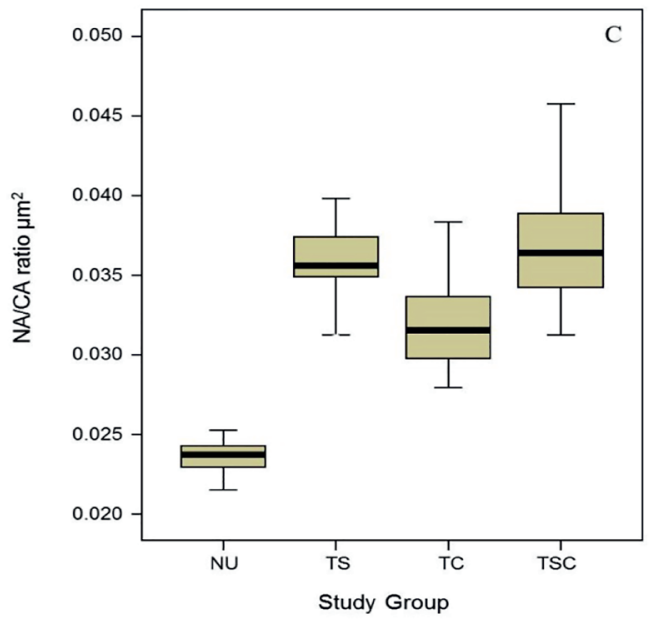

Figure 2. Boxplots for study groups: (A) For cytoplasmic area (CA); (B) For nuclear area (NA); (C) For nuclear/cytoplasmic area (NA/ $\mathrm{CA}$ ) ratio (NU: non users; TS: tobacco smoking; TC: tobacco chewing; TSC: tobacco smoking and chewing). 
Table II. Comparison of mean cytoplasmic area (CA) values, nuclear area (NA), nuclear and cytoplasmic area (NA/CA) of study groups.

$\begin{array}{lc}\text { A. Comparison of mean cytoplasmic area (CA) values of study groups } \\ \text { Study Group } & \left.\text { Cytoplasmic Area (Mean } \mu \mathrm{m}^{2} \pm \mathrm{SD}\right) \\ \text { Non users (NU) } & 4133.06 \pm 122.76 \\ \text { Tobacco smoking (TS) } & 3642.64 \pm 95.09 \\ \text { Tobacco chewing (TC) } & 3559.67 \pm 113.81 \\ \text { Tobacco smoking and chewing (TSC) } & 3336.96 \pm 163.87 \\ \text { B. Comparison of mean nuclear area (NA) values of study groups } \\ \text { Study Group } & \text { Nuclear Area (Mean } \mu^{2} \pm \mathrm{SD} \text { ) } \\ \text { Non users (NU) } & 97.44 \pm 5.91 \\ \text { Tobacco smoking (TS) } & 130.58 \pm 4.65 \\ \text { Tobacco chewing (TC) } & 114.26 \pm 6.18 \\ \text { Tobacco smoking and chewing (TSC) } & 122.22 \pm 5.83 \\ \text { C. Comparison of mean nuclear and cytoplasmic area (NA/CA) ratio values of study groups } \\ \text { Study Group } & \left.\text { NA/CA ratio (Mean } \mu \mathrm{m}^{2} \pm \mathrm{SD}\right) \\ \text { Non users (NU) } & 0.0236 \pm 0.00098 \\ \text { Tobacco smoking (TS) } & 0.0359 \pm 0.0022 \\ \text { Tobacco chewing (TC) } & 0.0322 \pm 0.0028 \\ \text { Tobacco smoking and chewing (TSC) } & 0.0368 \pm 0.0032 \\ \text { SD: Standard Deviation } & \end{array}$

ANOVA (analysis of variance) showed a significant effect for CA and NA in all habit groups with leukoplakia. There was a significant difference (by LSD procedure, $P=0.05)$ in the mean value of CA between the control group and tobacco groups. There was also a statistically significant $(P<0.05)$ reduction in $\mathrm{CA}$ from smokers to chewers, and mixed habit groups, respectively. A significant $(P<0.001)$ difference was found in CA of smokers and chewers. The mean NA values showed a statistically significant increase (by LSD procedure, $P=0.05$ ) in NA for all habit groups with leukoplakia as compared to non-users. There was also a statistically significant $(P<0.001)$ difference for NA between the habit groups.

The mean NA/CA ratio values showed a statistically significant increase (by LSD procedure, $P=0.05$ ) in NA/ $\mathrm{CA}$ ratio for all habit groups with leukoplakia as compared with non-users (Table IIC). There was also a statistically significant difference for NA/CA ratio between the habit groups, except between smoking and combined habit group $(P=0.225)$. There was no significant difference (by independent samples t-test, $P=0.05$ ) in the cytoplasmic area and the nuclear area between men and women for the control group.

\section{Discussion}

Epidemiological and clinical studies suggest a causative role of tobacco use in the evolution of oral precancerous and cancerous lesions. Leukoplakia is the most frequent premalignant lesion of the oral cavity [20]. Association of leukoplakia and tobacco chewing has been demonstrated in prospective studies as well as in casecontrol studies [21]. Prevalence of leukoplakia in regular smokeless tobacco users ranges from $3.5 \%$ to $22 \%$ [22]. Risk of oral leukoplakia is 4-6 times higher in smokers than non-smokers [20]. Tobacco associated leukoplakia becomes even more complicated in cases of mixed habits of tobacco chewing and smoking [23,24].

In the present study, there was a predominance of the male population over the female in all the groups. Male dominance can be related to cultural characteristics. Men generally practice tobacco chewing habits early in their adolescence and continue their habits until old age. There was no significant difference (by independent samples t-test, $P=0.05$ ) in the cytoplasmic area and the nuclear area between men and women for the control group. Cowpe et al. in his study considered that the gender of the patient does not influence the cellular or nuclear diameter of normal mucosal cells [25]. According to previous studies, there is no significant difference in cell and nuclear size over 40 years of age (26-28). So all the individuals in this study were over 40 years of age.

The use of alcohol with tobacco has been considered as an essential risk factor for oral cancer [29,30]. Alcohol drinking with tobacco use has a synergistic effect on the risk of oral cancer. So, subjects with alcohol habit were excluded from this study. The cellular changes have been observed in oral epithelial cells of tobacco users as early malignant changes [31,32]. The epithelium from both tobacco smoking and chewing individuals showed an increase in cell proliferation as compared to the nonuser of tobacco [3].

The decrease in the cell diameter and increase in the nuclear diameter are two significant morphologic changes required for replication that occur in actively proliferating cells. In the beginning, there is a reduction cell ability to differentiate into its more mature cell. Moreover, the cells related to the nucleoplasm reduces the amount of its cytoplasm. So the cell diameter decreases and the nuclear 
size increases. This may be related to the rise in the nuclear contents required for replication. As a result, there is an increase in the nuclear to cytoplasmic ratio to an extreme degree [33].

In the present study, there was a significant reduction in the cytoplasmic area (CA) and an increase in the nuclear area (NA) of all habit groups with leukoplakia as compared to the control group. Einstein et al. reported a reduction in cellular diameter and increase in nuclear diameter in buccal squames of tobacco users in south Indian population [34]. Ramesh et al. also reported a significant decrease in cell size and a substantial increase in nuclear size from healthy cells to mucosal lesions with dysplasia and further dysplasia to squamous cell carcinoma [35]. Lavanya et al. reported similar findings from standard samples to lesions with a white patch and further from red areas to squamous cell carcinoma in reverse smokers [36].

Franklin et al. [37] in his research observed the reduction in cell diameter at the dysplastic stage. The cells became smaller in comparison to the size seen in carcinomas. There was an increase in nuclear size followed by dysplasia and squamous cell carcinoma in experimental lesions on hamster cheek pouch epithelium [37]. Shabana et al. also observed increase the nuclear area from the normal epithelium to traumatic keratosis, leukoplakia and their highest value in squamous cell carcinoma [38]. Cowpe et al. found that tissues undergoing malignant transformation typically showed a reduction in the cytoplasmic area before an increase in the nuclear area [25].

The nuclear/cytoplasmic ratio represents the significant changes that occur in the cell at a morphological level [17]. Alterations in nuclear/cytoplasmic ratio are included among the criteria for dysplastic changes [8]. In the present study, there was a significant increase in the nuclear/cytoplasmic area ratio of all habit groups with leukoplakia as compared to the control group. Hillman et al. reported a higher nuclear/cytoplasmic ratio in heavier smokers [39]. Bharath et al. observed a significant increase in mean nuclear/cytoplasmic ratio from standard samples through lesions to squamous cell carcinoma in reverse smokers [36]. Cowpe et al. [25] also found considerable elevation in mean nuclear/cytoplasmic ratio of the abnormal oral mucosal squamous cell. This was due to a significant increase in the mean nuclear area and a reduction in the mean cytoplasmic area [25].

In the present study, the mean cytoplasmic area was lowest in the combined habit group followed by chewing group and smoking group. The mean nuclear area was highest in the smoking group, followed by combined habit group and chewing group.

Hence mean nuclear/cytoplasmic area ratio was highest in combined habit group followed by the smoking group and chewing group. This aim that the combined habit of smoking and chewing causes predisposes oral mucosa to oral cancer as compared to a single habit.
These cytomorphometric changes may be due to the effect of tobacco (smoking and chewing) on epithelial cells and its relation in the development of oral premalignant and malignant lesions $[13,35]$. The reduction in cellular diameter and increase in nuclear diameter can be an early indication of malignant changes, and this reflects the dysplastic changes taken place in premalignant lesions as compared to the normal oral mucosa. So, the premalignant lesions can be considered an intermediate stage in the range from normal to carcinoma [36].

The cellular changes occur at the molecular level during the transformation of healthy tissue to premalignancy or malignancy, before they are seen under the microscope and much before clinical changes become evident. Identification of high-risk oral premalignant lesions and intervention at premalignant stages can play an important key role in reducing mortality, morbidity and cost of oral squamous cell carcinoma associated treatment [40].

This study has assessed cytomorphometrical changes associated with the effect of tobacco use in leukoplakia and the results show that the alterations observed in the exfoliated buccal squamous of tobacco users are similar to those noted in histopathological sections of premalignant lesions resulted from the increased cellular activity. Therefore, this study supports the cause-effect relationship between tobacco use and oral precancer and cancer. The study has a limitation of a smaller sample size. Since the tobacco habits are prevalent in a large population, further studies are required with a larger sample size.

\section{Conclusion}

Cytomorphometric changes can be the earliest indicator of cellular and nuclear alterations. These changes suggest the influence of tobacco on the oral epithelium from normal to dysplasia, which can be useful for early indication of progression to the premalignant lesion. Exfoliative cytology, along with cytomorphometric analysis, can be of value for monitoring clinically suspicious lesions for early detection of malignancy. Exfoliative cytology can be of value as an adjunct to biopsy and also helps in the determination of the best site for biopsy for widespread white patches.

\section{References}

1. Krishna Priya M, Srinivas P, Devaki T. Evaluation of the Prevalence of Oral Mucosal Lesions in a Population of Eastern Coast of South India. J Int Soc Prev Community Dent. 2018;8:396-401.

2. Niaz K, Maqbool F, Khan F, Bahadar H, Ismail Hassan F, Abdollahi M. Smokeless tobacco (paan and gutkha) consumption, prevalence, and contribution to oral cancer. Epidemiol Health. 2017;39:e2017009.

3. Kamath VV, Anigol P, Setlur K. Micronuclei as prognostic indicators in oral cytological smears: A comparison between 
smokers and non-smokers. Clin Cancer Investig J. 2014;3:4954.

4. Muthukrishnan A, Warnakulasuriya S. Oral health consequences of smokeless tobacco use. Indian J Med Res. 2018;148:35-40.

5. Hernandez BY, Zhu X, Goodman MT, Gatewood R, Mendiola $\mathrm{P}$, Quinata $\mathrm{K}$, et al. Betel nut chewing, oral premalignant lesions, and the oral microbiome. PLoS One. 2017;12:e0172196.

6. Jairajpuri ZS, Rana S, Hajela A, Jetley S. Toward early diagnosis of oral cancer: Diagnostic utility of cytomorphological features, a pilot study. Natl J Maxillofac Surg. 2019;10:20-26.

7. Candau-Alvarez A, Dean-Ferrer A, Alamillos-Granados FJ, Heredero-Jung S, García-García B, Ruiz-Masera JJ, et al. Verrucous carcinoma of the oral mucosa: an epidemiological and follow-up study of patients treated with surgery in 5 last years. Med Oral Patol Oral Cir Bucal. 2014;19:e506-e511.

8. Seifi S, Feizi F, Mehdizadeh M, Khafri S, Ahmadi B. Evaluation of cytological alterations of oral mucosa in smokers and waterpipe users. Cell J. 2014;15:302-309.

9. Vineis P, Caporaso N. Tobacco and cancer: epidemiology and the laboratory. Environ Health Perspect. 1995;103:156-160.

10. Ghose UR, Parida BB. Cytological study of exfoliated buccal mucosa cells of tribes in Orissa State (India) with high risk for oral cancer. Indian J Cancer. 1995;32:95-99.

11. Al-Abbadi MA. Basics of cytology. Avicenna J Med. 2011;1:18-28.

12. Verma R, Singh A, Badni M, Chandra A, Gupta S, Verma R. Evaluation of exfoliative cytology in the diagnosis of oral premalignant and malignant lesions: A cytomorphometric analysis. Dent Res J (Isfahan). 2015;12:83-88.

13. Mendes SF, de Oliveira Ramos G, Rivero ER, Modolo F, Grando LJ, Meurer MI. Techniques for precancerous lesion diagnosis. J Oncol. 2011;2011:326094.

14. Raman RK, Kamboj M, Narwal A. The Diagnostic Role of Methyl Green-Pyronin Y Staining in Oral Leukoplakia and Oral Squamous Cell Carcinoma: An Exfoliative Cytology-Based Cytomorphometric Analysis. Acta Cytol. 2019;63:401-410.

15. Ogden GR, Cowpe JG, Green MW. The effect of distant malignancy upon quantitative cytologic assessment of normal oral mucosa. Cancer. 1990;65:477-480.

16. Lam VK, Nguyen TC, Chung BM, Nehmetallah G, Raub CB. Quantitative assessment of cancer cell morphology and motility using telecentric digital holographic microscopy and machine learning. Cytometry A. 2018;93:334-345.

17. Sahay K, Rehani S, Kardam P, Kumra M, Sharma R, Singh N. Cytomorphometric analysis and morphological assessment of oral exfoliated cells in type 2 diabetes mellitus and healthy individuals: A comparative study. J Cytol. 2017;34:27-33.

18. Singh M, Sircar K, Tandon A, Chowdhry A, Popli DB. The role of tobacco as an etiological agent for oral cancer: Cytomorphometrical analysis of the buccal mucosa in tobacco users. Dent Res J (Isfahan). 2014;11:649-655.

19. Warnakulasuriya S, Reibel J, Bouquot J, Dabelsteen E. Oral epithelial dysplasia classification systems: predictive value, utility, weaknesses and scope for improvement. J Oral Pathol Med. 2008;37:127-133.

20. Freitas MD, Blanco-Carrión A, Gándara-Vila $P$, Antúnez-López J, García-García A, Gándara Rey JM. Clinicopathologic aspects of oral leukoplakia in smokers and nonsmokers. Oral Surg Oral Med Oral Pathol Oral Radiol Endod. 2006;102:199-203.

21. Chaudhry K. Is pan masala-containing tobacco carcinogenic? Natl Med J India. 1999;12:21-27.

22. Chandra P, Govindraju P. Prevalence of oral mucosal lesions among tobacco users. Oral Health Prev Dent. 2012;10:149153.

23. van der Waal I, Schepman KP, van der Meij EH, Smeele LE. Oral leukoplakia: a clinicopathological review. Oral Oncol. 1997;33:291-301.

24. Chaturmohta A, Umarji HR, Kadam S, Nayyar AS, Puri H, Ayesha $\mathrm{H}$, et al. Evaluation of tobacco-associated oral lesions in the police personnel from greater Mumbai: A survey with review of literature. Adv Hum Biol. 2018;8:112-116.

25. Cowpe JG, Longmore RB, Green MW. Quantitative exfoliative cytology of normal oral squames: an age, site and sex-related survey. J R Soc Med. 1985;78:995-1004.

26. Ogden GR, Leigh I, Chisholm DM, Cowpe JG, Lane EB. Exfoliative cytology of normal oral mucosa. Assessing the basal cell keratin phenotype. Acta Cytol. 1996;40:933-936.

27. Miller SC, Soberman A, Stahl SS. A study of the cornification of the oral mucosa of young male adults. J Dent Res. 1951;30:4-11.

28. Pappelis GA, Pappelis AJ, Courtis WS. Nuclear dry mass and area variations in human buccal mucosa cells. Acta Cytol. 1973;17:37-41.

29. Khan Z, Dreger S, Shah SMH, Pohlabeln H, Khan S, Ullah Z, et al. Oral cancer via the bargain bin: The risk of oral cancer associated with a smokeless tobacco product (Naswar). PLoS One. 2017;12:e0180445.

30. Rivera C. Essentials of oral cancer. Int J Clin Exp Pathol. 2015;8:11884-11894.

31. Goud ML, Mohapatra SC, Mohapatra P, Gaur SD, Pant GC, Knanna MN. Epidemiological correlates between consumption of Indian chewing tobacco and oral cancer. Eur J Epidemiol. 1990;6:219-222.

32. Godavarthy D, Naik R, Mujib BR, Gali PK, Smitha V, Nair M. Tobacco-induced alterations in exfoliated oral epithelial cells: A comparative image analysis study. J NTR Univ Health Sci. 2018;7:168-173.

33. Demaria S, Pikarsky E, Karin M, Coussens LM, Chen YC, El-Omar EM, et al. Cancer and inflammation: promise for biological therapy. J Immunother. 2010;33:335-351.

34. Einstein TB, Sivapathasundharam B. Cytomorphometric analysis of the buccal mucosa of tobacco users. Indian J Dent Res. 2005;16:42-46.

35. Ramaesh T, Mendis BR, Ratnatunga N, Thattil RO. Cytomorphometric analysis of squames obtained from normal oral mucosa and lesions of oral leukoplakia and squamous cell carcinoma. J Oral Pathol Med. 1998;27:83-86. 
36. Bharath TS, Kumar NG, Nagaraja A, Saraswathi TR, Babu GS, Raju PR. Palatal changes of reverse smokers in a rural coastal Andhra population with review of literature. J Oral Maxillofac Pathol. 2015;19:182-187.

37. Franklin CD, Smith CJ. Stereological analysis of histological parameters in experimental premalignant hamster cheek pouch epithelium. J Pathol. 1980;130:201-215.

38. Shabana AH, el-Labban NG, Lee KW. Morphometric analysis of basal cell layer in oral premalignant white lesions and squamous cell carcinoma. J Clin Pathol. 1987;40:454-458.

39. Hillman RW, Kissin B. Oral cytologic patterns in relation to smoking habits. Some epithelial, microfloral, and leukocytic characteristics. Oral Surg Oral Med Oral Pathol. 1976;42:366-374.

40. Giovannacci I, Magnoni C, Vescovi P, Painelli A, Tarentini E, Meleti M. Which are the main fluorophores in skin and oral mucosa? A review with emphasis on clinical applications of tissue autofluorescence. Arch Oral Biol. 2019;105:89-98. 